\title{
Objective Beach-State Classification From Optical Sensing of Cross-Shore Dissipation Profiles
}

\author{
Matthew Browne, Darrell Strauss, Rodger Tomlinson, and Michael Blumenstein
}

\begin{abstract}
Remote sensing using terrestrial optical chargecoupled device cameras is a useful data collection method for geophysical measurement in the nearshore zone, where in situ measurement is difficult and time consuming. In particular, optical video sensing of the variability in human-visible surface refraction due to the nearshore incident wave field is becoming an established method for distal measurement of nearshore subtidal morphology. We report on the use of a low-mounted shore-normal camera for gathering data on cross-shore dissipative characteristics of a dynamic open beach. Data are analyzed for the purposes of classifying three of Wright and Shorts' intermediate classes of morphological beach state as determined by expert raters. Although these beach states are usually thought of as being distinctive in terms of their longshore bar variability, theory predicts that differences should also be observed in cross-shore dissipative characteristics. Three methods of generating features from statistical features from the archived optical data are described and compared in terms of their ability to discriminate between the beach states. Principal component scores of the percentile distributions were found to provide slightly better classification performance (i.e., $85 \%$, while approximating the data using relatively fewer features), whereas classification using intensity distributions alone resulted in the worst performance, classifying $78 \%$ of beach states correctly. Class center moment profiles for each beach state were constructed, and results indicate that cross-shore wave dissipation becomes more disorganized as linear bars devolve into more complex transverse structures.
\end{abstract}

Index Terms-Beach state, classification, nearshore, optical.

\section{INTRODUCTION}

$\mathbf{U}$ NDERSTANDING the nearshore beach zone represents a significant challenge for researchers, due to the intrinsic complexity of sediment and fluid dynamics in such a highenergy environment. Complex bathymetric features that arise through processes of sediment erosion and accretion are highly significant in the study of coastal processes. Transformation and dissipation of the incident wave field as it interacts with subsurface bathymetry result in changes in surface reflectance in the human-visible spectrum, due to the generation of foam (white water) and shadows as waves shoal. It is therefore possible to infer the existence of subsurface bathymetric characteristics from distal measurements of water surface reflectance in the presence of shoaling and breaking waves [3], [9], [12].

The difficulties associated with the direct collection of longterm morphological data in highly dynamic nearshore zones has resulted in a great deal of interest in the use of remote

Manuscript received July 18, 2005; revised February 1, 2006. This work was supported by the Australian Research Council.

The authors are with CSIRO Mathematical and Information Sciences, Cleveland 4163, Australia (e-mail: matthew.browne@csiro.au).

Digital Object Identifier 10.1109/TGRS.2006.877758 sensing methods. Shore-mounted optical cameras have become an established instrument for gathering data related to the morphology in the seabed nearshore zone [7]. Aarninkhof and Ruessink [8] provide a review of the variety of shore-based, airborne, optical, or penetrative radar and spectral instruments that have been applied in monitoring the surf zone. The most significant body of work using this approach is grounded in the Argus system [3], [9] for the purpose of quantifying intermediate beach states, and significant work has established the general validity of inferring bathymetric characteristics from average surface reflectance.

The basic approach is to measure the average surface water reflectance in the break zone and to average over a period $>10 \mathrm{~min}$. Zones of high average reflectance are associated with wave breaking, rollers, and residual foam (white water) and are related to areas of relatively shallow water (i.e., sandbars). It was possible for Lippmann and Holman [3] to use this monitoring system for further defining the relationship between environmental parameters and Wright and Short's intermediate beach states. A large body of work has established that average surface reflectance intensity is related to various bathymetric properties. For example, video-derived observations have served for sandbar localization [3], [9], [10], with Kingston et al. [10] using an empirical neural network method to correct inferred cross-shore sandbar maxima from pixel intensity profiles. Aarninkhof et al. [11] estimated nearshore bathymetry from Argus images using a process-based inverse model of wave-breaking dynamics, achieving acceptable predictions for breaker parameters within the range for which the model had been calibrated.

Although it is known that average surface reflectance is related to subtidal morphology, it is recognized that it is an imperfect measurement technique. Wave height and quality create different patterns of dissipation that vary independently with the morphology. This approach is necessarily limited when swell conditions are insufficiently large to cause significant breaking. In such cases, outer bars may be present but are undetectable because breaking is not evident. The cases of storms and large swell conditions are also problematic, as the large quantity of residual surface foam results in a serious lack of definition in the detected bars. In general, foam residue generated from wave breaking may remain on the water surface, biasing the estimate of the bar location [8], [9]. Recent work has focused on methods to minimize the impact of surface foam for linking pixelintensity-based dissipation with wave dissipation derived from theoretical models [12]. Wave dissipation maxima, and that of pixel intensity, were found to somewhat reflect the greatest rate of depth change rather than absolute depth [13]. 


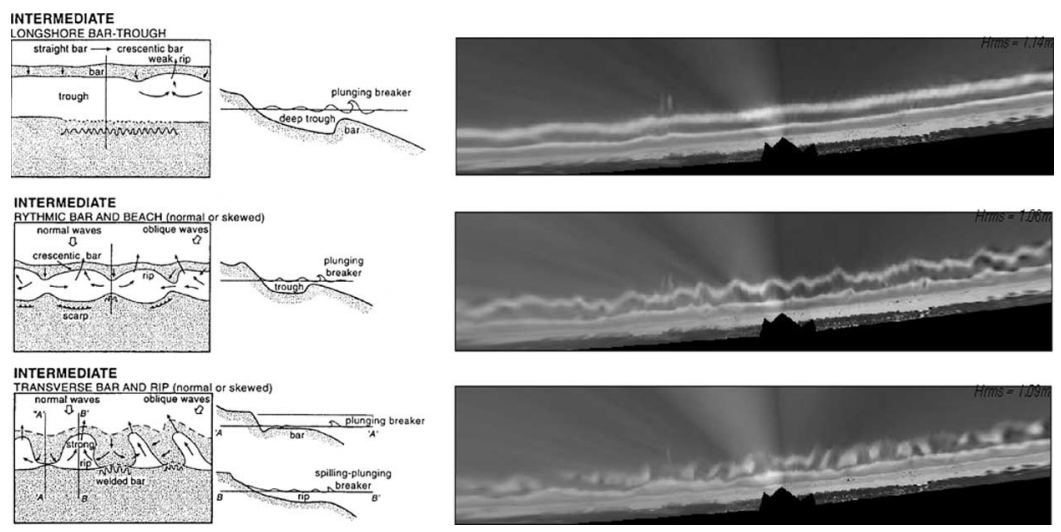

Fig. 1. Wright and Short intermediate beach states commonly observed at Narrowneck beach. (Left) Cross-shore and longshore theoretical dynamics and (right) Corresponding longshore Argus images from representative days in the survey period. The intermediate states of interest are LBT, RBB, and TBR. The Argus images were used as a basis for human expert judgement of beach state.

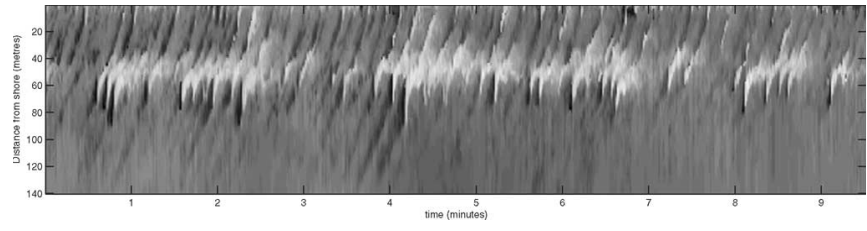

Fig. 2. Example of a cross-shore intensity timestack from the Narrowneck camera. The diagonal patterns represent individual waves propagating onshore. Note that different cross-shore locations (horizontal slices) are characterized by a particular combination of shoaling, breaking, and persistent white-water effects.

A major application of the time-averaged surface reflectance sensing method has been in the validation of dynamic models of beach evolution. The classification of individual beach states most often follows Wright and Short's approach [1]-[3] (Fig. 1), which is based on variations in the underwater geophysical morphology. Derived from a long-term data set of beach surveys, the scheme involves a range of six observed states varying from dissipative, through intermediate, to reflective. Wright and Short's categorical scheme incorporates predictions regarding the sequential progression of beach states under various swell conditions and involves descriptions of both the longshore and cross-shore variabilities in morphology. Thus, the model attempts to describe qualitatively the evolution of nearshore morphological features in terms of their scale and shape in response to environmental energy input over time, mainly in the form of incident waves [3]-[6]. Accurate estimation of beach state, either by remote sensing or by in situ human experts (e.g., lifeguards), is an oft-performed and essential task for determining public safety for beach use and in estimating beach health for planning nourishment programs.

The present study makes the proposition that higher order statistics (i.e., variance, skewness, and kurtosis) of surface reflectance may better capture the total range of wave dissipation/ transformation behavior and therefore provide more information regarding the underlying morphology. Fig. 2 provides a useful "timestack" representation of cross-shore wave dissipation over time. The progress of individual waves may be observed as each progresses through the stages of shoaling and breaking at the main sandbar (approximately $60 \mathrm{~m}$ offshore). If horizontal cross-shore slices are considered, it is clear that different cross-shore locations correspond to different patterns of surface reflectance over time. For example, wave shoaling around $90-110 \mathrm{~m}$ offshore results in a steeper wave face and creates shadows, leading to darker registered pixel values [14]. Inasmuch as large waves occur only intermittently, this would be characterized by a degree of skewness in the crossshore reflectance measure. In the main breaking zone $(60-80 \mathrm{~m}$ offshore), high variance in the reflectance values would be observed. The empirical work presented in subsequent sections involves characterizing the cross-shore dissipation/ transformation profile in a manner that incorporates higher order/non-Gaussian characteristics and examining the relationship of this fingerprint to beach state.

As well as predicting differences in longshore bar variability across the three categories, Wright and Short's model makes predictions regarding the form of cross-shore dissipation profile that ought to be observed. Thus, with reference to Fig. 1, the model predicts that the longshore bar and trough (LBT) state will be characterized by a well-defined bar formation and a deep trough to shoreward. This would be expected to generate wave transformation and dissipation activity well localized with respect to cross-shore position. The rhythmic bar and beach (RBB) state, with a more shallow trough and more poorly defined bar, should lead to a less organized dissipation activity and rollers traversing a greater extent of the zone shoreward of the bar. Finally, the transverse bar and rip (TBR) state, with the least degree of bar definition and an almost flat profile in the break zone, should generate a mixture of spilling and plunging waves over the entire extent of the dissipation zone.

A significant shortcoming of many of the previous studies in this area is that they have relied on subjective identification of beach states [15]. Lippmann and Holman [3] relied on a large number (i.e., nine) of expert raters in an attempt to remove this subjectivity, whereas Wright et al. [6] and, more recently, Ranasinghe et al. [15] are the only studies that report a statistical discrimination between beach states. The most encouraging result reported was that of a $90 \%$ agreement between visual classification and discriminate model predictions, using a measure of longshore bar variability as a predictor [15]. Objective discrimination of beach state is an important component of computational systems for monitoring the nearshore zone [16]. 


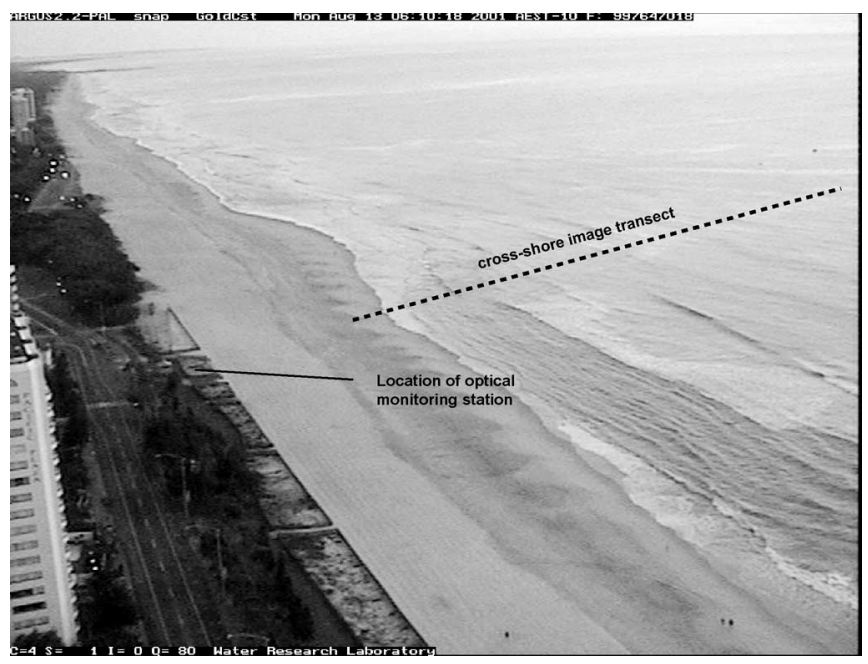

Fig. 3. Study site at Narrowneck beach at the Gold Coast, Australia.

In this work, we propose the use of more sophisticated representations of variability in cross-shore surface reflectance as an alternative to the conventional approach of using the average reflectance. We evaluate the effectiveness of this extra information in terms of classification of beach state in terms of Wright and Short's model (Fig. 1).

\section{DATA}

Data were collected from a shore-normal low-angle camera. Although this does not provide a complete longshore perspective, this view angle is conducive to examination of variations in cross-shore bar morphology in the surf zone. It has added advantages in that it is portable, cost effective, and does not require the use of a special high-altitude mounting site.

Video data were collected from Narrowneck beach (see Fig. 3), located north of Surfers Paradise at the northern end of the Gold Coast, Queensland. Narrowneck beach has been studied particularly intensively in recent years because it is the site of an artificial reef constructed by the local government authorities.

Median sediment size at Narrowneck is $0.2-0.22 \mathrm{~mm}$ [17]. The tidal range is classed as microtidal, and tidal amplitudes vary up to $2.1 \mathrm{~m}$ with a mean range of $1 \mathrm{~m}$. Sand is generally transported within a $15-\mathrm{m}$ depth of water with the limit of offshore and onshore transport being 22 and $30 \mathrm{~m}$, respectively. Seabed profiles obtained during bathymetric surveys prior to construction of Narrowneck reef show a steep beach face and either: 1) a nearly horizontal beach platform or channel merging with an offshore bar or 2) a well-developed channel and bar system. The profile is nearly linear further offshore with a gradient of approximately $1: 50$. There is usually only one or two breakpoint longshore bars, but their cross-shore position varies considerably [18].

Average deep-water wave heights generally range from 0.8 to $1.4 \mathrm{~m}$ with periods of 7-9 s [19]. Extreme events such as cyclones can produce offshore wave heights up to $14 \mathrm{~m}$ and wave periods up to $18 \mathrm{~s}$ [20]. Typically, although waves over $1.5 \mathrm{~m}$ will result in the formation of a storm bar along Gold
Coast beaches, the reflective extreme of the Wright and Short [2] beach-state model is seldom observed.

A fixed remote digital video camera was installed at a height of approximately $15 \mathrm{~m}$ above the high-tide mark and $30 \mathrm{~m}$ south of the southernmost point of the artificial reef, with an orientation due east and a downward angle of approximately $10^{\circ}$. The optical sensor used red, blue, and green (RBG) filters in the standard visual range, and these bands were averaged to create a monochrome representation of total reflectance in the visible spectrum. Digital video recordings are made and archived on the hour in daylight hours for the duration of $10 \mathrm{~min}$ and with a pixel resolution of $240 \times 320$, sampled at $2 \mathrm{~Hz}$, with a median geographical resolution of $0.92 \mathrm{~m}$. Video data began archiving in December 2004, and the current report describes analysis of data gathered up to March 2005. After discarding video records due to salt accretion on the camera lens, bad weather, and low lighting conditions, 284 records were available for analysis.

A cross-shore section in the center of the visual field was extracted from each 10-min video record and rectified to as to be linearly scaled in the cross-shore direction. A spatial resolution of $1 \mathrm{~m}$ was settled upon, and the index to the cross-shore location was determined from the mean high-tide water level. The cross-shore transect was sampled from 1 to $140 \mathrm{~m}$ from this point and recorded for 1200 time samples $(2 \mathrm{~Hz} \times 60 \mathrm{~s} \times 10 \mathrm{~min})$. Each record thus comprises a $1200 \times$ 140 two-dimensional (2-D) timestack $I(t, m)$ of intensities $I$ at each relative time instant $t$ and cross-shore location $m$. Each record was histogram equalized to include an approximately equal distribution of reflectance intensities over the domain $(t, m)$. This was found to be helpful in standardizing records over varying light conditions and emphasizes the "relative" reflectance at each location $(t, m)$ for subsequent feature extraction. Fig. 4 (left) shows an example of a histogram-normalized record.

Corresponding Argus images of Narrowneck beach for each of the days recorded were scrutinized by three human experts and were classified as to belonging to one of the three intermediate Wright and Short beach states. This entailed that manual classification of the records was performed using the highaltitude time-averaged images generated by the Argus system as a reference rather than the video gathered in the present study. Thus, the data used as the source for predictor (independent) and target predicted (dependent) variables were from independent sources. Inasmuch as Argus images provide a clear view of bar contours in the longshore, the degree of observed longshore variability observed by the expert rater was the main criteria for state classification. The video data collected in this study provided a high-resolution cross-shore section with information on the cross-shore dissipation profile rather than longshore variability. Thus, one of the issues addressed concerns whether or not it is possible to determine the intermediate state of the beach solely from the cross-shore dissipative wave behavior. The average interrater agreement was $88 \%$, which is high relative to degrees of human interrater agreement reported in previous studies of beach-state classification. A beach-state classification consensus was generated by a majority voting system, whereby the final class was determined by the majority classification 

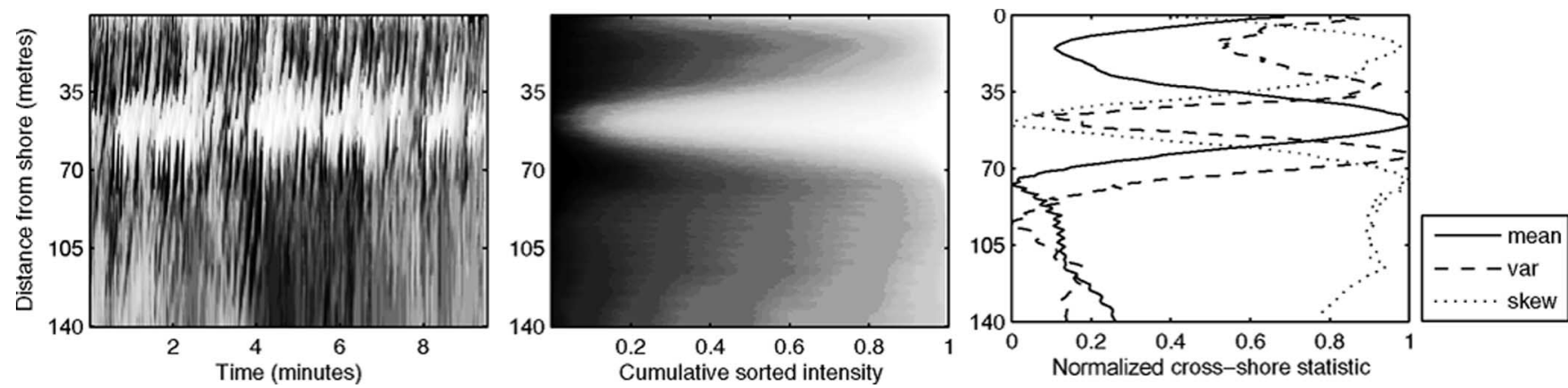

Fig. 4. (Left) Histogram-equalized timestack may be transformed via (center) sorting or (right) calculation of moments, both operations performed with respect to rows. In doing so, time information is discarded, but the overall variability of the intensity over time is retained.

where there was a $2: 1$ ratio between the expert judgements. The issue of a $1: 1: 1$ expert rater split did not arise.

\section{ANALYSIS}

The data preprocessing and analysis stages are summarized as follows:

1) rejection of poor-quality records and expert classification (described in Section II);

2) generation of shore-normal, rectified, and histogramequalized transect slices and creation of cross-shore timestack records (Section II);

3) transformation to three candidate cross-shore dissipation profile representations of the statistical distribution, in increasing order of quantity of information retained, i.e., intensities, moments, and sorted intensities (described below);

4) dimensionality reduction to create a small number of input features, using principal components analysis (PCA) (below);

5) classification of three classes using linear discriminant analysis (LDA) (below).

The total data set $I^{k}(t, m)(k=1, \ldots, 284)$ was transformed to three different forms of representation before subsequent dimensionality reduction and inclusion in an LDA. The term "distribution" in this paper is used through to refer to the statistical pattern of intensity observations over time. One goals of this paper is to compare several methods of representing the statistical distribution of intensities. Each of the following representations of the cross-shore intensity distribution were tested.

Average intensities: The standard method of averaging pixel intensities over $t$, implemented to serve as a baseline comparison case for the other methods. There are 140 element description $(1 \times 140$ cross-shore positions). This feature representation of the dissipation activity corresponds to an average of the rows of $I^{k}(t, m)$. In the example provided in Fig. 4, this corresponds to the solid line in the right-hand side subplot. The peak around $70 \mathrm{~m}$ from the shore indicates the location of the main break zone associated with the linear bar.

Distribution moments: This represents an extension of the average intensity profile to include higher moments; variance, skewness, and kurtosis are calculated with respect to $t: 560$ element description (4 distribution moments $\times 140$ cross-shore positions). This feature representation of the dissipation activity corresponds to the calculation of higher moments of the inten- sities in each of the rows of $I^{k}(t, m)$. In the example provided in Fig. 4, these data correspond to the solid, dashed, and dotted lines in the right-hand side subplot. The peak in the variance plot around $80 \mathrm{~m}$ from the shore indicates the location that most peaks shoal and break, generating a mixture of intermittent dark shadows and light white water.

Intensity percentiles: The most complete representation of the distribution of optical intensities over time: sorting $I^{k}(t, m)$ with respect to $t$. For each cross-shore position, intensity values measured over time are sorted (thus discarding timeorder information). This is equivalent to an inverse cumulative probability distribution. The sorting procedure discards timeorder information but loses no other information regarding the statistical distribution of intensities over time and is therefore the most complete descriptor of stochastic distribution of the data. This "intensity percentile" representation involved the creation of down-sampled 2-D feature map of $I^{k}(t, m): 1400$ element description ( 20 percentiles $\times 70$ cross-shore positions, percentiles and cross-shore pixels sampled at a lower rate to maintain reasonable data size). In the example provided in Fig. 4, these data correspond to the center subplot. The sorted rows are essentially inverse empirical probability distribution functions, retaining all statistical information without assuming a Gaussian distribution.

Dimensionality reduction was necessary to reduce each individual video record to a feature vector that provided suitable input to a statistical classifier system. The method of linear PCA ${ }^{1}$ has long been employed as a method of concisely describing cross-shore bathymetry surveys [21]-[23] and is a standard procedure for dimensionality reduction [24]. ${ }^{2}$ There are many possible extensions of simple linear PCA, ${ }^{3}$ and nonlinear and

\footnotetext{
${ }^{1}$ Also described as empirical orthogonal functions (EOF) or independent components analysis (ICA) in the literature.

${ }^{2}$ By determining the principal eigenvalues of the correlation matrix of a data set, PCA may be used for feature extraction and dimensionality reduction. Projection of the original data onto the eigenfunctions results in scores formed by an orthogonal rotation of the original axes to align with the principal axes of variation (eigenfunctions). At any reduction in dimensionality (discarding axes that describe proportionally little variance), PCA scores may be said to be an optimal description of the original data in a linear orthogonal least squares sense. Dommenget and Latif [25] note that the shape of PCA eigenfunctions is strongly determined by the orthogonality constraint, and hasty visual interpretation is inadvisable.

${ }^{3}$ By relaxing the conditions of linearity or orthogonality, or substituting some other (i.e., curved or circular) geometric coordinate system for that of straight lines, or performing PCA on local regions of a coordinate space, or by some combination of the above.
} 

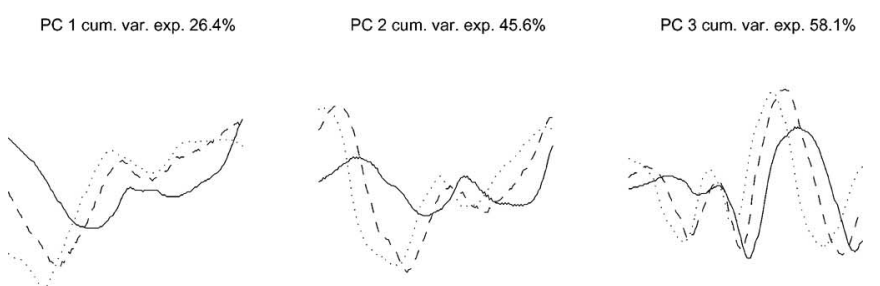

PC 4 cum. var. exp. $65.0 \%$

PC 5 cum. var. exp. $70.8 \%$
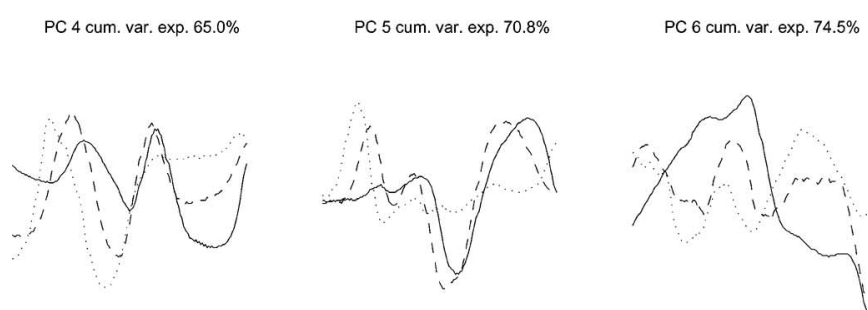

PC 7 cum. var. exp. $78.0 \%$

PC 8 cum. var. exp. $80.8 \%$

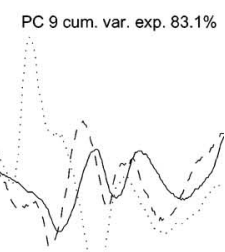

(a)
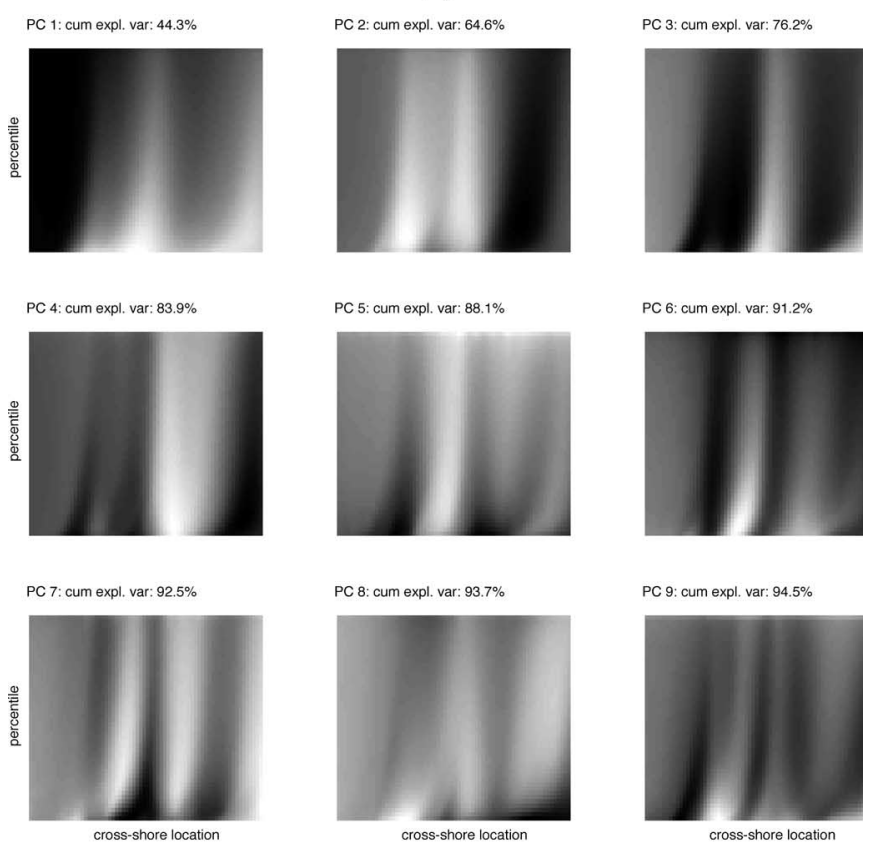

(b)

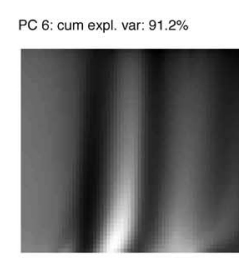

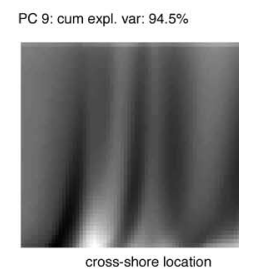

Fig. 5. First nine principal components of two cross-shore dissipation representations, with cumulative explained variance. In the example given in Fig. 4, these basis functions were used to transform the sorted cross-shore intensity values on the center and right subplots to obtain a low-dimensional feature vector. Note that the axes are reversed. (a) Solid, dashed, and dotted lines indicate principal components for mean, variance, and skewness, respectively (kurtosis curve omitted for clarity). (b) PCA-derived 2-D percentile/order versus distance-from-shore image of the principal components for the percentile (sorted) intensity basis functions.

complex variants have been reported to produce more efficient state descriptors of nearshore bathymetry in circumstances where the underlying dynamic structure is nonlinear or periodic

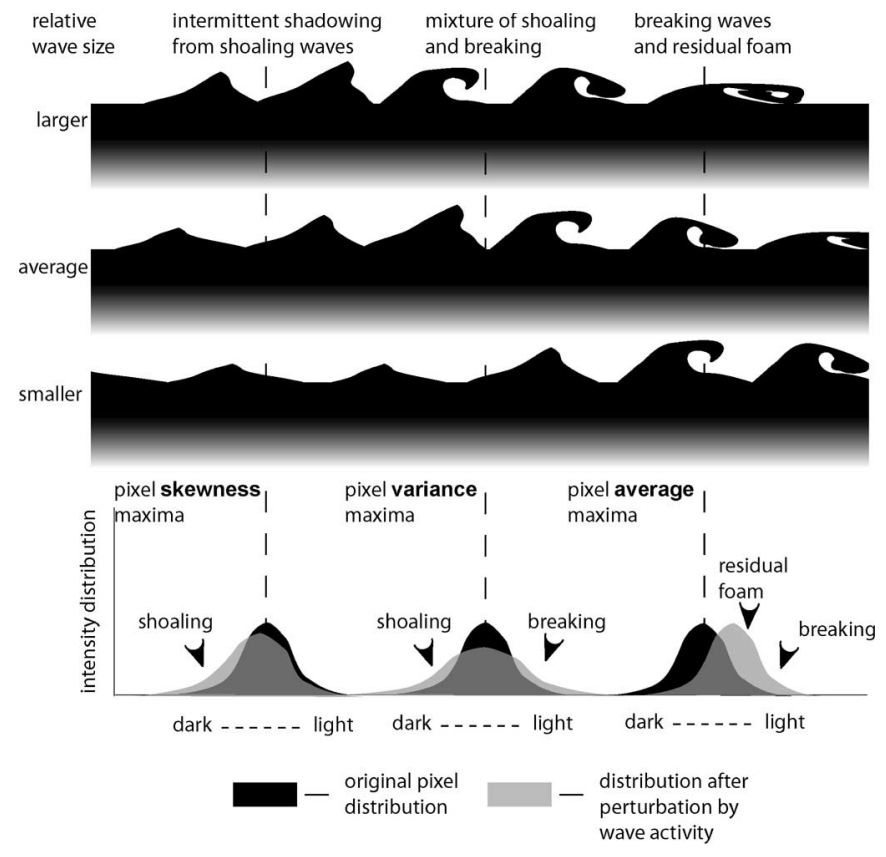

Fig. 6. Dynamics of wave transformation and dissipation for variable swell in the short term leads to complex effects on the observed pixel distributions.

[23]. Before applying more sophisticated forms of PCA, a good practical approach seems to be to apply more sophisticated variants if, upon inspection, there appears to be residual nonlinear structure in the scatter plots of the derived scores.

Fig. 5(a) shows the first nine principal components identified for the cross-shore moment profiles. Fig. 5(b) illustrates the derived principal components of the percentile matrices as 2-D images. Note that in each of these plots, the cross-shore location denoted on the $x$ axis runs from seaward (left) to shoreward (right). In Fig. 5(a), it should be emphasized that the derived components represent the joint moments of the distribution. Thus, correlations between moments at particular cross-shore positions may be considered by the PCA algorithm. Similarly, in Fig. 5(b), the principal components may take into account regularities between different percentile/cross-shore locations.

Examination of Fig. 5(a) reveals that the peaks of each of the moment curves generally proceed from higher to lower, as one moves shoreward. For example, a typical sequence is a peak skew value to seaward, followed by a peak variance value, which in turn precedes the peak mean value. We explain this as a consequence of the dynamics of wave dissipation and short-term variability of swell size and water level (e.g., due to infragravity waves). As Fig. 6 illustrates using three example break progressions, normal variability in swell and water level will lead to variability in the observed breaking dynamics. For example, a larger swell that approaches the shore during the trough of an infragravity wave will shoal and break at a shallower depth. Thus, for a given static bathymetry, and at any particular distance from the shore, there is some variability as to what stage of transformation/dissipation the waves will be in. Next, due to the intermittent nature of wave groups (we observe that over a period of time, a pixel value is perturbed only about $10 \%$ of the time), the effect of swell on an observed baseline 


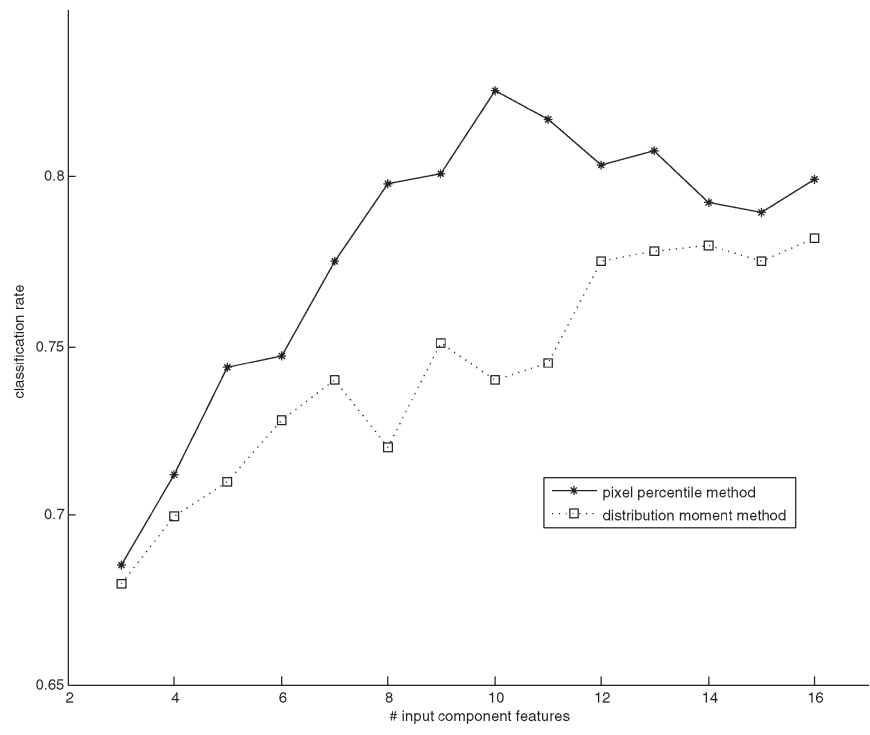

Fig. 7. Comparison of classification rates of logistic regression using PCA of pixel percentiles (sorted values) and distribution moments (mean, variance, etc.) using 1-16 principal components.

pixel distribution (i.e., what is observed in the remaining $90 \%$ of the time) would not be expected to be a simple shifting of the mean. A more accurate view would be that wave activity produces a mixture of Gaussian distributions, with a "waveperturbed" pixel distribution overlying the base probabilities of observing particular intensity values. An exception to this rule is the existence of persistent white-water foam, which is an indirect outcome of wave breaking and may be expected to partially or completely replace the base distribution itself. Finally, waves produce different effects on the observed pixel value at different stages in transformation/dissipation progression; darker values are observed during shoaling, whereas lighter values are observed after breaking. As Fig. 6 illustrates, the expected combined result of these interacting effects agrees with that observed experimentally on waves breaking on a typical bar formation: a perturbation of the observed pixel distribution that is dominated, in order, by skewness, variance, and mean, as one moves shoreward.

In Fig. 5(b), a subtle diagonal shoreward curve may be observed on many of the basis functions, there being a diagonal relationship between low percentiles and the higher percentiles at a slightly more shoreward location. This phenomenon may be explained once it is recognized that the distribution of wave heights is itself Gaussian: The shoaling-breaking progression of relatively infrequent large waves (represented in the lower percentiles) breaks further out to sea, skewing the morphology of the basis functions in Fig. 5(b) to the left. Overall, there is a dependence across statistics and cross-shore position, due to the physical behavior in the system. For example, high variability at one cross-shore location (due to a mixture of peaking and breaking) is physically related to a higher mean at another slightly more shoreward cross-shore location (as the same waves break or create swash). The fact that there exists an interaction between percentile and cross-shore location somewhat supports the use of the combined matrix representation, as the linearly redundant information may be approximated more efficiently by combined (percentile, cross-shore position) eigenfunctions.

\section{RESUlts}

Logistic LDA, which implements a linear discriminant function suitable for categorical output [24], [26], was implemented for classifying beach state. LDA is a straightforward linear classification technique closely related to linear regression. Linear decision boundaries were determined between each of the states using the reduced dimension feature vector generated by projection of the optical intensity representations onto the basis functions derived by the PCA procedure. The target for the classifier was the human-expert-determined classes, namely LBT, RBB, and TBR.

As described in the previous section, PCA was applied to three approaches representing the gathered optical intensity data: referred to as "average intensities," "moments," and "sorted intensities." It should be recalled that because the moments method incorporates mean intensities (along with variance, skewness, and kurtosis), the information contained in the average representation is a subset of this representation. Preliminary analysis found that the average intensities representation resulted in a peak classification performance of $61 \%$, significantly less than the other approaches. With this in mind, the results presented below focus on the other two more comprehensive descriptors of the cross-shore dissipation profile.

Fig. 7 compares classification rates on the validation set for the cross-shore percentile method and the moments method. Performance under the null hypothesis condition (that the classifier performs no better than chance) for such a three-class classification task would be $33.3 \%$. The 284 video records were divided ten times into $90 \%$ training and $10 \%$ test sets, each time reserving a different portion of the data for testing in a rotating design.

Using a feature vector generated from the percentile representation results in the best classification performance, of $83 \%$ on the validation subset using the first ten principal component scores as input. As more than ten components are included, the classification rate begins to decline due to overfitting and subsequent lack of generalization of the model. From Fig. 7, it may be seen that the discriminate performance of the classifier using a feature vector based on the normalized cross-shore moments rises more slowly than the percentile approach as the number of feature inputs are increased. This result is consistent with the fact that the sorting approach to representing distributions of intensities retains more information than the moment approach.

The resulting classification performance on the test sets using ten principal components are presented in Table I, which shows the confusion matrix for the classifiers between the three beach states. As mentioned in Section II, the average interrater agreement between the human experts was $88 \%$. Thus, the observed performance of the automatic classifier (at $83 \%$ ) was quite close to the level of agreement between the human experts.

Fig. 8 plots the first three rectified moment profiles of the class centers of each of the three intermediate beach states. Some interpretation of the derived profiles with respect to 
TABLE I

LDA ClassificATION RATES With TEN PRINCIPAL Components of CRoss-Shore Percentiles

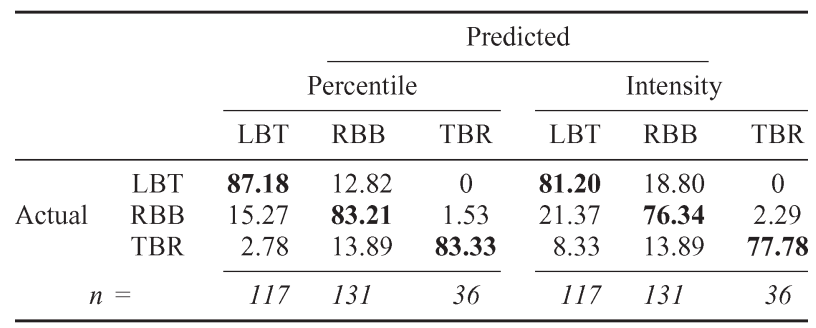

Wright and Short's description of the beach states is noted in the figure.

\section{CONCLUSION}

Despite the fact that video-based sensing of the nearshore zone is fast becoming the standard data gathering technique in coastal processes research, there has been relatively little attention paid to developing automatic and objective methods of quantification and classification. The classification rates achieved in this study were encouraging and on a par with the degree of agreement between human raters. This degree of reliability helps demonstrate the feasibility of objective determination of beach state from low-mounted video sensors and supports the predictions of Wright and Short's model in terms of differences between the cross-shore dissipative profiles of the three states.

As expected, the most complete method of extracting the parameters of a distribution, i.e., the percentile position representation, achieved better classification rates than either the intensity or moment representations, although utilizing fewer extracted features to do so. Naturally, the modest sample size and the relative differences in performance do not permit strong conclusions to be drawn regarding the merits of the three feature extraction methods tested. However, the relative strength of the percentile approach is consistent with our assumptions that the cross-shore intensity distribution at a particular cross-shore location will not necessarily be well described by a single Gaussian distribution. Also, the intensity distributions consist of subdistributional elements correlated with elements at other cross-shore locations. This is evidenced in Fig. 5(b) in the small but significant degree of curved diagonal features in the derived components. Thus, intensity values, and also higher moments, would not be expected to be the most efficient method of describing this form of data. The percentile method, because it makes no assumptions regarding the underlying shape of the pixel intensity distribution, appears to be more efficient at representing the observed activity. However, the disadvantage of this approach is that components extracted from the distributions are less interpretable. Thus, for the purposes of illustrating graphically the differences between the beach states in Fig. 8, scaled and rectified moment profiles are more suitable for discussion.

The results provide another source of support for the validity of Wright and Short's categories of beach state, at least in terms of the distinction between the intermediate states. Human perception, even that of experts with scientific training, is well
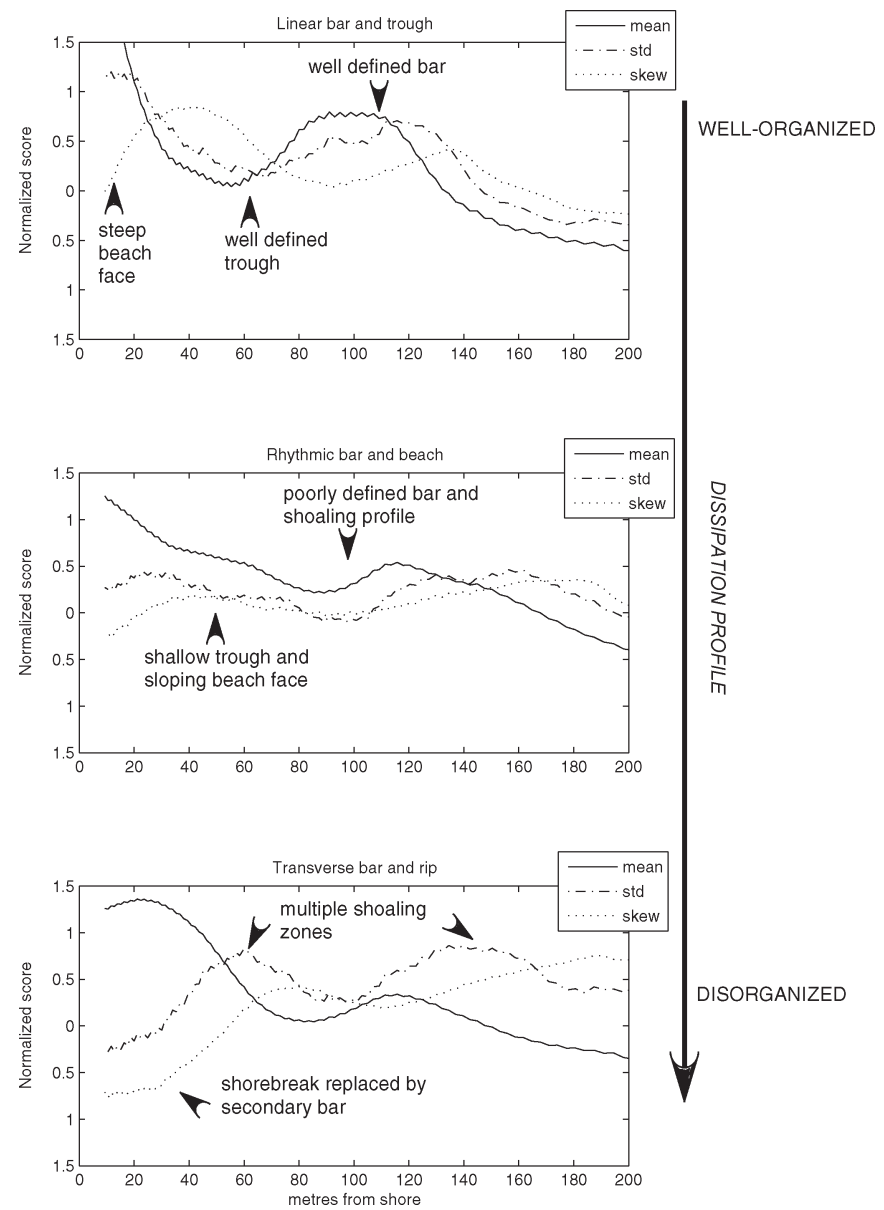

Fig. 8. Moment profiles (mean, standard deviation, and variance with respect to the number of meters from the shore) of the derived class centers of the three beach states. Comments specify the features that appeared to represent a significant basis for the discriminate function.

known for its susceptibility to bias and systematic error, and objective methods for quantification of video data have long been recognized as desirable in the marine geophysics community [9]. Thus, a high degree of consistency between human and machine classifiers supports the beach-state classification scheme, which hitherto has been mainly supported by data derived from human observations. The derived rectified class centers of the three beach states shown in Fig. 8 display decreasing linear dependence on cross-shore position and less well defined bar and trough formation, as beach state evolves from the LBT class to more complex bar formations. Also observed was a decreasing degree of coherence between the various moments, indicating that the classic skew-variance-mean maxima progression breaks down as beach state devolves from a simple bar-trough formation to more complex transverse structures.

The feature extraction approach pursued in this paper has been variously described as assumption free or "data driven"the point being that rather than specifying the features of interest, the domain of interest is specified (i.e., the cross-shore pixel intensity statistics), and an unsupervised approximation method (i.e., PCA) is used to derive features for classification. There are a number of advantages and disadvantages associated with this approach; on one hand, the derived features are almost certainly suboptimal, whereas, on the other hand, the 
blind variance maximization criterion can discover features difficult for an experimenter to define a priori. Perhaps the greatest disadvantage is the difficulty of interpreting the derived discriminate model, although this is mitigated by the fact that pixel intensity values themselves do not have a straightforward interpretation in terms of subtidal bathymetry [13]. It may be that future research should continue to concentrate on modeling the relationship between nearshore dissipation and bathymetry before attempting to directly estimate beach state.

Limitations and concerns notwithstanding, the classification rate achieved and the differences observed in the posthoc plots of the class centers indicate that there appears to be sufficient information contained in the cross-shore dissipation profile to discriminate intermediate beach states. The Wright and Short beach-state model, which has recently had support in terms of its predictions of longshore bar variability [15], has been supported in this study in terms of predictions regarding crossshore dissipation. With the development of an appropriate data processing methodology, low-mounted shore-normal cameras may have the potential to provide useful information regarding subtidal bathymetry and beach state.

\section{ACKNOWLEDGMENT}

The authors would like to thank C. Lane of CoastalWatch for assistance in gathering data for this paper.

\section{REFERENCES}

[1] L. D. Wright and A. D. Short, "Morphodynamics of beaches and surf zones in Australia," in Handbook of Coastal Processes and Erosion. Boca Raton, FL: CRC, 1983, pp. 35-64.

[2] - "Morphodynamic variability of surf zones and beaches: A synthesis," Mar. Geol., vol. 56, no. 1-4, pp. 93-118, Apr. 1984.

[3] T. Lippmann and R. Holman, "The spatial and temporal variability of sand bar morphology,” J. Geophys. Res., vol. 95, no. C7, pp. 11 575-11590, Jul. 1990.

[4] J. Chappell and I. G. Eliot, "Surf-beach dynamics in time and space-An Australian case study, and elements of a predictive model," Mar. Geol., vol. 32, no. 3-4, pp. 231-250, Jul. 1979.

[5] L. D. Wright, A. D. Short, and M. O. Green, "Short term changes in the morphodynamic states of beaches and surf zones: An empirical predictive model," Mar. Geol., vol. 62, no. 3-4, pp. 339-364, Jan. 1985.

[6] L. D. Wright, A. D. Short, J. D. Boon, III, B. Hayden, S. Kimball, and J. H. List, "The morphodynamic effects of incident wave groupiness and tide range on an energetic beach," Mar. Geol., vol. 74, no. 1-2, pp. 1-20, Jan. 1987.

[7] K. T. Holland, R. A. Holman, T. C. Lippmann, J. Stanley, and N. Plant, "Practical use of video imagery in nearshore oceanographic field studies," J. Ocean. Eng., vol. 22, no. 1, pp. 81-92, Jan. 1997.

[8] S. Aarninkhof and B. Ruessink, "Video observations of wave breaking and the implication for wave decay modelling," in Proc. Coastal Dyn. Conf., Lund, Sweden, 2001, pp. 979-988.

[9] T. Lippman and R. Holman, "Quantification of sand bar morphology: A video technique based on wave dissipation," J. Geophys. Res., vol. 94, no. C1, pp. 995-1011, Jan. 1989.

[10] K. Kingston, B. Ruessink, I. van Enckevort, and M. Davidson, "Artificial neural network correction of remotely sensed sandbar location," Mar. Geol., vol. 169, no. 1, pp. 137-160, Sep. 2000.

[11] S. Aarninkhof, P. Janssen, and N. Plant, "Quantitative estimations of bar dynamics from video images," in Proc. Coastal Dyn., Plymoth, U.K., 1997, pp. 365-374.

[12] S. G. J. Aarninkhof and B. G. Ruessink, "Video observations and model predictions of depth-induced wave dissipation," IEEE Trans. Geosci. Remote Sens., vol. 42, no. 11, pp. 2612-2622, Nov. 2004.

[13] I. M. J. van Enckevort and B. G. Ruessink, "Video observations of nearshore bar behaviour. Part 1: Alongshore uniform variability," Cont. Shelf Res., vol. 23, no. 5, pp. 501-512, Mar. 2003.
[14] H. F. Stockdon and R. A. Holman, "Estimation of wave phase speed and nearshore bathymetry from video imagery," J. Geophys. Res., vol. 105, no. C9, pp. 22 015-22 033, Sep. 2000.

[15] R. Ranasinghe, G. Symonds, K. Black, and R. Holman, "Morphodynamics of intermediate beaches: A video imaging and numerical modelling study," Coastal Eng., vol. 51, no. 7, pp. 629-655, Sep. 2004.

[16] M. Browne, M. Blumenstein, R. Tomlinson, and C. Lane, "An intelligent system for remote monitoring and prediction of beach conditions," in Proc. IASTED Int. Conf. Artif. Intell. and Appl., 2005, pp. 533-537.

[17] Hyder Consulting, Patterson Britton \& Partners and WBM Oceanics Australia, "Tweed River Entrance Sand Bypassing Project Permanent Bypassing System,” NSW Dept. Land Water Conserv. Qld Dept. Environ, Tech. Rep., 1997.

[18] J. A. Hutt, K. P. Black, and S. T. Mead, "Narrowneck reef. Report 1: Surf zone experiments. report prepared for GCCC by centre of excellence in coastal oceanography and marine geology," Univ. Waikato and NIWA, Hamilton, New Zealand, Tech. Rep. 1, 1998.

[19] L. A. Jackson, "Coastal engineering works - Gold Coast beach replenishment report," Gold Coast City Council, Nerang, Australia, Tech. Rep. $152,1992$.

[20] Environmental Services Division-Coastal Services, Coastal Services Monitor Storm Event-March 2004, Environ. Protection Agency, Queensland Gov. Tech. Rep., 2004.

[21] C. Winant, D. Inman, and C. Nordstrom, "Description of seasonal beach changes using empirical eigenfunctions," J. Geophys. Res., vol. 80, no. 15, pp. 1979-1986, 1975.

[22] K. Wijnberg and J. Terwindt, "Extracting decadal morphological behaviour from high-resolution, long-term bathymetric surveys along the holland coast using eigenfunction analysis," Mar. Geol., vol. 126, no. 126 , pp. 301-330, 1995 .

[23] B. Ruessink, I. van Enckevort, and Y. Kuriyama, "Non-linear principal component analysis of nearshore bathymetry," Mar. Geol., vol. 203, no. 1-2, pp. 185-197, Jan. 2004

[24] P. McCullagh and J. A. Nelder, Generalized Linear Models, 2nd ed. London, U.K.: Chapman \& Hall, 1989.

[25] D. Dommenget and M. Latif, "A cautionary note on the interpretation of EOFs," J. Clim., vol. 15, no. 2, pp. 216-225, Feb. 2002.

[26] B. D. Ripley, Pattern Classification and Neural Networks. Cambridge, U.K.: Cambridge Univ. Press, 1996.

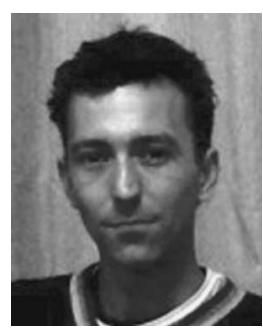

Matthew Browne received the Ph.D. degree on the use of wavelets for the analysis of evoked potentials in 2002 .

He has worked on postdoctoral projects in mobile autonomous robotics in Japan and Germany and has developed remote environmental monitoring systems in Australia for commercial implementation. He is currently a Research Scientist with the Division of Mathematical and Information Sciences, Commonwealth Science and Industry Research Organization, Cleveland, Australia. His current research interest is in modeling large and complex environmental data sets.

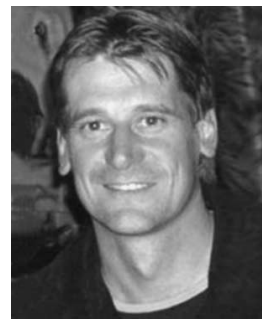

Darrell Strauss received the B.S. degree in meteorology and oceanography from Flinders University of South Australia, Adelaide, Australia, in 1992. $\mathrm{He}$ is currently working toward the Ph.D. degree at Griffith University, Brisbane, Australia.

From 1995 to 2004, he was with the National Tidal Facility, Australia. His current research interest is on the morphological modeling of intermediate beachstate transitions 


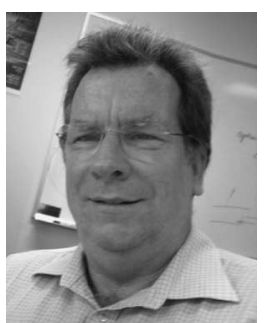

Rodger Tomlinson received the Civil Engineering degree and the Ph.D. degree from the University of New South Wales, Sydney, Australia, in 1977 and 1986, respectively.

He has been with Griffith University, Brisbane, Australia, since 1992 as the Head of the School of Environmental Engineering and since 2000 as the Foundation Director of the Griffith Center for Coastal Management based on the Gold Coast Campus. His current research interests concentrate on the planning and management for natural variability on open coastlines, which include historical and process studies of the impact of climate change and climate variability on coastlines, tidal entrances, and coastal waterways.

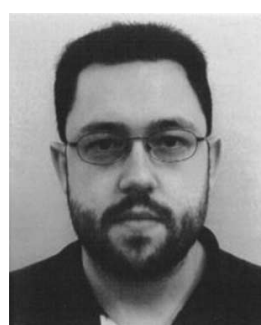

Michael Blumenstein received the B.S. degree in computing, the B.I.T. degree (with honors), and the Ph.D. degree from Griffith University, Brisbane, Australia, in 1996. 1997, and 2001, respectively.

$\mathrm{He}$ is currently with the School of Information and Communication Technology, Griffith University, where he is a Senior Lecturer and also the Deputy Head of School. His research interests include object detection and recognition from digital video imagery (including cluttered outdoor scenes), offline cursive handwriting recognition, and neural network applications in engineering, environmental science, and coastal management. He has published more than 40 papers in refereed conferences and journals in these areas. He has also supervised more than a dozen research students.

Dr. Blumenstein has been invited to serve as Organizing Chair, Program Chair, and Committee Member for a number of national/international conferences in his areas of expertise. 